\title{
Asymptotic Inequalities \\ Related to the Maximum Modulus of a Polynomial
}

\author{
C. Frappier and M. A. Qazi
}

\begin{abstract}
Let $\mathcal{P}_{n}$ be the class of all polynomials of degree at most $n$. If $\|\cdot\|$ denotes the supremum norm on $|z|=1$ and $M_{P}(R)=\max _{|z|=R}|P(z)|$, then for an arbitrary polynomial $P(z)=\sum_{\nu=0}^{n} a_{\nu} z^{\nu}$ in $\mathcal{P}_{n}$ the inequality $M_{P}(R) \leq R^{n}\|P\|$ holds, with equality if and only if $a_{0}=\ldots=a_{n-1}=0$. Given $n, k \in \mathbb{N}$ with $0 \leq k \leq n-1$, let $\varphi_{n, k}(R)$ be the largest number such that $M_{P}(R)+\varphi_{n, k}(R)\left|a_{k}\right| \leq R^{n}\|P\|(R \geq 1)$ for all $P \in \mathcal{P}_{n}$. Values of $\varphi_{n, k}(R)$ for $k=0$ and $k=1$ are known since some time. We study the case $k \geq 2$.
\end{abstract}

Keywords: Polynomials, inequalities, asymptotic

AMS subject classiflcation: $30 \mathrm{~A} 10,30 \mathrm{C} 10,26 \mathrm{D} 05$

\section{Introduction}

Let $\mathcal{P}_{n}$ be the class of polynomials $P(z)=\sum_{\nu=0}^{n} a_{\nu} z^{\nu}$ of degree at most $n$. We write

$$
\|P\|=\max _{|z|=1}|P(z)|
$$

and

$$
M_{P}(R)=\max _{|z|=R}|P(z)|
$$

If $P \in \mathcal{P}_{n}$, then according to a well-known result of S. Bernstein [1: p. 45],

$$
\left\|P^{\prime}\right\| \leq n\|P\|
$$

Also the inequality (see [7])

$$
M_{P}(R) \leq R^{n}\|P\| \quad(R \geq 1)
$$

C. Frappier: École Polytechn. de Montréal, Dép. de Math. et de Génie industriel, Campus de l'Univ., C.P. 6079, Succursale Centre-Ville Montréal (Québec), H3C 3A7

M. A. Qazi: École Polytechn. de Montréal, Dép. de Math. et de Génie industriel, Campus de I'Univ., C.P. 6079, Succursale Centre-Ville Montréal (Québec), H3C 3A7

Research was supported by the Natural Sciences and Engineering Research Council of Canada, Grant No. OGP0009331.

ISSN 0232-2064 $/ \$ 2.50$ (C) Heldermann Verlag 
is well known. In both inequalities (1.3) and (1.4) equality holds if and only if $P(z)=$ $a_{n} z^{n}$, i.e. if and only if the coefficients $a_{\nu}(\nu=0, \ldots, n-1)$ are zero. Given $n \in \mathbb{N}$ and $0 \leq k \leq n-1$, let $c_{k}(n)$ and $\varphi_{n, k}(R)$ be the best possible constants such that

$$
\left\|P^{\prime}\right\|+c_{k}(n)\left|a_{k}\right| \leq n\|P\|
$$

and

$$
M_{P}(R)+\varphi_{n, k}(R)\left|a_{k}\right| \leq R^{n}\|P\| \quad(R \geq 1)
$$

hold, respectively, for all $P \in \mathcal{P}_{n}$. In inequality (1.6) the statement "best possible" must be understood in the following sense:

For every $\varepsilon>0$ there exists $P_{\varepsilon} \in \mathcal{P}_{n}$ with $P_{\varepsilon}(z)=\sum_{\nu=0}^{n} a_{\nu}(\varepsilon) z^{\nu}$ such that

$$
M_{P_{\varepsilon}}(R)+\left(\varphi_{n, k}(R)+\varepsilon\right)\left|a_{k}(\varepsilon)\right|>R^{n}\left\|P_{\varepsilon}\right\| \text {. }
$$

An analoguous statement holds for inequality (1.5).

Exact values of the constants $c_{0}(n)$ and $c_{1}(n)$ have been calculated in [5]. The value of the constant $c_{2}(n)$ appears in [4]. Unfortunately determining the exact value of the constants $c_{k}(n)$ for $k \geq 3$ turns out to be quite cumbersome. However in [3] we were successful in investigating their asymptotic behaviour. To be precise we proved the following

Theorem A (see [3]). Let $c_{k}(n) \quad(0 \leq k \leq n-1)$ be the constants in inequality (1.5). For each $k$, the sequence $\left\{c_{k}(n)\right\}_{n \in \mathbb{N}}$ tends to a finite limit $c_{k}$ as $n \rightarrow \infty$. This limit constant $c_{k}$ is, for $k \geq 2$, the smallest positive root of the equation

$$
D(2, \underbrace{0,0, \ldots, 0}_{k \text { zeros }}, x,-2 x, x, \underbrace{0,0, \ldots, 0}_{k-2 \text { zeros }})=0
$$

where $D\left(b_{0}, b_{1}, b_{2}, \ldots, b_{n}\right)$ is the determinant of the matrix

$$
M\left(b_{0}, b_{1}, b_{2}, \ldots, b_{n}\right)=\left(\begin{array}{ccccc}
b_{0} & b_{1} & \cdots & b_{n-1} & b_{n} \\
\bar{b}_{1} & b_{0} & \cdots & b_{n-2} & b_{n-1} \\
\vdots & \vdots & \ddots & \vdots & \vdots \\
\bar{b}_{n-1} & \bar{b}_{n-2} & \cdots & b_{0} & b_{1} \\
\bar{b}_{n} & \bar{b}_{n-1} & \cdots & \bar{b}_{1} & b_{0}
\end{array}\right) .
$$

As regards the constants $\varphi_{n, k}(R) \quad(0 \leq k \leq n-1)$ in inequality (1.6), values of $\varphi_{n, 0}(R)$ and $\varphi_{n, 1}(R)$ are known since some time. Indeed we have the following

Theorem B (see [5: p. 70]). Let $P \in \mathcal{P}_{n}$ with $n \geq 2$. Then for all $R \geq 1$

$$
M_{P}(R)+\left(R^{n}-R^{n-2}\right)\left|a_{0}\right| \leq R^{n}\|P\| .
$$

The coefficient of $\left|a_{0}\right|$ is best possible for each $R$.

Theorem C (see [5: p. 80]). Let $R \geq 1$. Then

$$
M_{P}(R)+\varphi_{n, 1}(R)\left|a_{1}\right| \leq R^{n}\|P\| \quad \text { for all } P \in \mathcal{P}_{n}
$$


where

$$
\begin{aligned}
& \varphi_{1,1}(R)=0 \\
& \varphi_{2,1}(R)=R\left(\sqrt{\frac{R^{2}+1}{2}}-1\right) \\
& \varphi_{3,1}(R)=\left(R^{2}-R\right)\left(\sqrt{R^{2}+R+1}-1\right) \\
& \varphi_{n, 1}(R)=\left(R^{n}-R^{n-2}\right)\left(\sqrt{1+\frac{1}{R^{2}}}-\frac{1}{R}\right) \quad \text { for } n \geq 4 .
\end{aligned}
$$

The coefficient of $\left|a_{1}\right|$ is best possible for each $R$.

In this paper we study the constants $\varphi_{n, k}(R)(0 \leq k \leq n-1)$ in inequality (1.6) for $k \geq 2$.

\section{A method of proof}

The method of proof we wish to describe here has been used successfully to establish various extremal inequalities (in particular (1.7) and (1.8)). We include its details for sake of completeness.

Consider two analytic functions

$$
f(z)=\sum_{\nu=0}^{\infty} a_{\nu} z^{\nu} \quad \text { and } \quad g(z)=\sum_{\nu=0}^{\infty} b_{\nu} z^{\nu} \quad(|z| \leq 1)
$$

The function

$$
(f * g)(z)=\sum_{\nu=0}^{\infty} a_{\nu} b_{\nu} z^{\nu} \quad(|z| \leq 1)
$$

is said to be their Hadamard product. Let us denote by $\mathcal{B}_{n}$ the subclass of $\mathcal{P}_{n}$ consisting of those polynomials $Q$ for which

$$
\|Q * P\| \leq\|P\| \quad \text { for all } P \in \mathcal{P}_{n} .
$$

To any polynomial $Q \in \mathcal{P}_{n}$ we associate the polynomial $\widetilde{Q}(z)=z^{n} \overline{Q\left(\frac{1}{z}\right)}$; thus $\widetilde{Q}$ depends on the class $\mathcal{P}_{n}$ and not just on $Q$. Observe the equivalence

$$
Q \in \mathcal{B}_{n} \quad \Longleftrightarrow \quad \tilde{Q} \in \mathcal{B}_{n} .
$$

In looking for the constants $\varphi_{n, k}(R)$ in (1.6) we divide both sides of this inequality by $R^{n}$ and note that

$$
\frac{1}{R^{n}}\left(M_{P}(R)+\varphi_{n, k}(R)\left|a_{k}\right|\right)=\sup _{|\alpha|<\varphi_{n, k}(R)}\|Q * P\|
$$

where

$$
Q(z)=Q_{\alpha}(z):=\sum_{k \neq j=1}^{n} \frac{R^{j} z^{j}}{R^{n}}+\frac{R^{k}+\bar{\alpha}}{R^{n}} z^{k}
$$


Then it would suffice to show that $Q_{\alpha} \in \mathcal{B}_{n}$ if $|\alpha|<\varphi_{n, k}(R)$. In view of the equivalence (2.2) we may prove, instead, that $\widetilde{Q}_{\alpha} \in \mathcal{B}_{n}$ if $|\alpha|<\varphi_{n, k}(R)$. We shall do the latter since $\widetilde{Q}_{\alpha}(0)=1$ and a fairly straightforward procedure described below can be used.

Let us denote by $\mathcal{B}_{n}^{0}$ the subclass of $\mathcal{B}_{n}$ consisting of those polynomials $P$ in $\mathcal{B}_{n}$ for which $P(0)=1$. The following lemma contains a useful characterization of $\mathcal{B}_{n}^{0}$.

Lemma 1 [5: Lemma 2]. The polynomial $\sum_{j=0}^{n} b_{j} z^{j}$ with $b_{0}=1$ belongs to $\mathcal{B}_{n}^{0}$ if and only if the matrix $M\left(1, b_{1}, b_{2}, \ldots, b_{n}\right)$ is positive semidefinite.

In order to study the definiteness of the matrix $M\left(1, b_{1}, b_{2}, \ldots, b_{n}\right)$ associated with the polynomial $\widetilde{Q}(z)=1+\sum_{j=0}^{n} b_{j} z^{j}$ the following well-known result is used.

Lemma 2 [6: Vol. 1/p. 337]. The Hermitian matrix

$$
\left(\begin{array}{cccc}
a_{11} & a_{12} & \cdots & a_{1 n} \\
a_{21} & a_{22} & \cdots & a_{2 n} \\
\vdots & \vdots & \ddots & \vdots \\
a_{n 1} & a_{n 2} & \cdots & a_{n n}
\end{array}\right) \quad \text { with } a_{i j}=\bar{a}_{j i}
$$

is positive definite if and only if its leading principal minors are all positive.

According to Lemma 1 , the inequality $\left\|\tilde{Q}_{\alpha} * P\right\| \leq\|P\|$ is satisfied for all $P \in \mathcal{P}_{n}$ and $|\alpha| \leq \varphi_{n, k}(R)$ if and only if the matrix

$$
M\left(R^{n}, R^{n-1}, \ldots, R^{k+1}, R^{k}+\alpha, R^{k-1}, \ldots, R^{2}, R, 1\right)
$$

is positive semidefinite for $|\alpha| \leq \varphi_{n, k}(R)$. Now by Lemma 2 , the constant $\varphi_{n, k}(R)$ can be seen as the largest number $\xi_{n, k}(R)$ such that each leading principal minor of the matrix (2.3) is positive for all $\alpha$ in $|z|<\xi_{n, k}(R)$. The positivity of the leading principal minors of order less or equal $n-k$ does not depend on $\alpha$ and is easily checked. Indeed, this follows in the light of the above discussion and from the fact that inequality (1.4) holds. Thus only the leading principal minors of order greater or equal $n-k+1$ of the matrix (2.3) need to be investigated. However, determining the exact value of $\xi_{n, k}(R)$, i.e. $\varphi_{n, k}(R)$ when $3 \leq k \leq n-1$ turns out to be complicated. In this paper we will study its behaviour as $R \rightarrow \infty$. To be precise we will prove the following Then

Theorem. Let the constants $\varphi_{n, k}(R)(0 \leq k \leq n-1)$ be as in inequality (1.6).

$$
\varphi_{n, k}(R)=\left(R^{n}-R^{n-2}\right)\left(1-f_{k}(R)\right) \quad \text { for } 2+2 k \leq n
$$

where $0 \leq f_{k}(R) \leq 1$ is independent of $n$. Furthermore

$$
\lim _{R \rightarrow \infty} R f_{k}(R)=2 \cos \frac{\pi}{k+2} \quad(k \geq 0)
$$




\section{Auxiliary results}

For the proof of the above theorem, some auxiliary results will be needed. They are presented in this section.

Lemma 3. Let $0 \leq j \leq k \leq n$ and set

$$
d_{n, k, j}(R ; \alpha)=D\left(R^{n}, R^{n-1}, \ldots, R^{k+1}, R^{k}+\alpha, R^{k-1}, \ldots, R^{j}\right) \quad(\alpha \in \mathbb{C}) .
$$

Then

$$
d_{n, k, j}(R ; \alpha) \equiv R^{n-j+1} d_{n-1, k-1, j-1}\left(R ; \frac{\alpha}{R}\right)
$$

The proof of the above Lemma is straightforward. Applying (3.2) recursively we immediately obtain

Corollary 1. If $0 \leq j \leq k \leq n$, then

$$
d_{n, k, j}(R ; \alpha) \equiv R^{j(n-j+1)} d_{n-j, k-j, 0}\left(R ; \frac{\alpha}{R^{j}}\right) .
$$

As another consequence of Lemma 3 we mention

Corollary 2. If $n \geq k$, then

$$
\varphi_{n, k}(R) \leq R^{n}-R^{n-2} \quad(R \geq 1) .
$$

Proof. It follows directly from Lemma 3 that

$$
\varphi_{n, k}(R) \leq R \varphi_{n-1, k-1}(R) \quad \text { for } 1 \leq k \leq n .
$$

Now applying (3.4) recursively and using Theorem B we get (3.3) for $n \geq k+2$. The cases $n=k+1$ and $n=k$ are obvious since $\varphi_{1,0}(R)=R-1$ and $\varphi_{0,0}(R)=0$

Our next lemma is a crucial one in the study of the asymptotic behaviour of the constants $\varphi_{n, k}(R)$ in inequality (1.6).

Lemma 4. Let $0 \leq j \leq k \leq n$ and $d_{n, k, j}(R ; \alpha)$ the function defined in (3.1). Then for $R \geq 1$ the roots $\lambda$ of the equation

$$
d_{n, k, j}(R ; \lambda)=0
$$

are real.

Proof. The proof is analogous to that of [3: Lemma 6] and it is thus omitted 


\section{Proof of the Theorem}

Let us recall from Section 2 that in order to study the constants $\varphi_{n, k}(R)(0 \leq k \leq n-1)$ in inequality (1.6) we are led to examine the positivity of the leading principal minors of order greater or equal $n-k+1$ of the matrix (2.3). In view of Corollary 1 we may instead consider the determinant

$$
D\left(R^{m}, R^{m-1}, \ldots, R^{s+1}, R^{s}+\gamma, R^{s-1}, \ldots, R^{2}, R, 1\right)
$$

where $m=n-j, s=k-j$ and $\gamma=\frac{\alpha}{R^{\prime}}$. By means of appropriate elementary operations and substitutions we will reduce this latter determinant to one which is simpler to handle and which will eventually lead to the desired result. The following reasoning may need to be adapted if $s=0, s=1$ or $s=2$.

So take the determinant (4.1) and

(i) subtract $\frac{1}{R}$ times its $(i+1)$-st row from the $i$-th row for $i=1,2, \ldots, m$. Then

(ii) for $i=1,2, \ldots, m$ divide the $i$-th row by $R^{2}-1$.

(iii) In the resulting determinant subtract $\frac{1}{R}$ times the $(l+1)$-st column from the $l$-th one for $l=1,2, \ldots, m$.

(iv) Now note that for $m \geq 2 s+2$ each of the rows number $s+2$ to $m-s-1$ of the resulting determinant contains only one non-zero element and that it is $R^{m-2}$ (nothing has to be done if $m=2 s+2$ ). Developping it by these $m-2 s-2$ rows one after the other yields the equality

$$
\begin{aligned}
D\left(R^{m}, R^{m-1}\right. & \left.\ldots, R^{s+1}, R^{s}+\gamma, R^{s-1}, \ldots, R^{2}, R ; 1\right) \\
& =R^{(m-2)(m-2 s-2)}\left(R^{2}-1\right)^{m} \mid\left[\left.c_{\mu, \nu}\right|_{1 \leq \mu, \nu \leq 2 s+3}\right.
\end{aligned}
$$

where

$$
\begin{aligned}
c_{\mu, \nu}=\left\{\begin{array}{ll}
R^{m-2} & \text { if } \nu=\mu \\
-\frac{\gamma}{R\left(R^{2}-1\right)} & \text { if } \nu=\mu+s+1 \\
\frac{\left(R^{2}+1\right) \gamma}{R^{2}\left(R^{2}-1\right)} & \text { if } \nu=\mu+s+2 \\
-\frac{\gamma}{R\left(R^{2}-1\right)} & \text { if } \nu=\mu+s+3 \\
0 & \text { otherwise }
\end{array} \quad \text { (for } 1 \leq \mu \leq s\right) \\
c_{s+1, \nu}= \begin{cases}R^{m-2} & \text { if } \nu=s+1 \\
-\frac{\gamma}{R\left(R^{2}-1\right)} & \text { if } \nu=2 s+2 \\
\frac{\gamma}{R^{2}-1} & \text { if } \nu=2 s+3 \\
0 & \text { otherwise }\end{cases} \\
c_{\mu, \nu}=\left\{\begin{array}{ll}
-\frac{\bar{\gamma}}{R\left(R^{2}-1\right)} & \text { if } \nu=\mu-s-3 \\
\frac{\left(R^{2}+1\right) \bar{\gamma}}{R^{2}\left(R^{2}-1\right)} & \text { if } \nu=\mu-s-2 \\
-\frac{\bar{\gamma}}{R\left(R^{2}-1\right)} & \text { if } \nu=\mu-s-1 \\
R^{m-2} & \text { if } \nu=\mu \\
0 & \text { otherwise }
\end{array} \quad \text { (for } s+2 \leq \mu \leq 2 s+2\right)
\end{aligned}
$$


(here, and in what follows, there is no term if $\nu \leq 0$ ) and

$$
c_{2 s+3, \nu}= \begin{cases}-\frac{\bar{\gamma}}{R} & \text { if } \nu=s \\ \bar{\gamma} & \text { if } \nu=s+1 \\ R^{m} & \text { if } \nu=2 s+3 \\ 0 & \text { otherwise. }\end{cases}
$$

(v) In equality (4.2) put $\gamma=\beta\left(R^{m}-R^{m-2}\right)$. Next

(vi) divide the $i$-th row by $R^{m-4}$ for $i=1,2, \ldots, 2 s+3$. Then factorize $R$ from columns $s+1, s+2$ and $2 s+3$. We follow by factorizing $R$ from rows $s+1, s+2$ and $2 s+3$. The determinant so obtained is easily seen to be an expression of the form $F\left(|\beta|^{2}\right)$ where $F$ is a polynomial; thus its minimum is independent of $\arg \beta$. Indeed, simply multiply the first $s+1$ columns by $\beta$ and then divide the first $s+1$ rows by the same quantity. Furthermore it is independent of $n$. To be precise, if we divide the last row by $R^{2}-1$, then

$$
\begin{aligned}
D\left(R^{m}, R^{m-1}\right. & \left.\ldots, R^{s+1}, R^{s}+\gamma, R^{s-1}, \ldots, R^{2}, R, 1\right) \\
& =R^{m^{2}-m-4 s-2}\left(R^{2}-1\right)^{m+1}\left|\left[c_{\mu, \nu}^{\prime}\right]\right|_{1 \leq \mu, \nu \leq 2 s+3}
\end{aligned}
$$

where, with $x:=|\beta|$,

$$
\begin{aligned}
& c_{1, \nu}^{\prime}= \begin{cases}R^{2} & \text { if } \nu=1 \\
-1 & \text { if } \nu=s+2 \\
R^{2}+1 & \text { if } \nu=s+3 \\
-R & \text { if } \nu=s+4 \\
0 & \text { otherwise }\end{cases} \\
& c_{\mu, \nu}^{\prime}=\left\{\begin{array}{ll}
R^{2} & \text { if } \nu=\mu \\
-R & \text { if } \nu=\mu+s+1 \\
R^{2}+1 & \text { if } \nu=\mu+s+2 \\
-R & \text { if } \nu=\mu+s+3 \\
0 & \text { otherwise }
\end{array} \quad \text { for } 2 \leq \mu \leq s-1\right) \\
& c_{s, \nu}^{\prime}= \begin{cases}R^{2} & \text { if } \nu=s \\
-R & \text { if } \nu=2 s+1 \\
R^{2}+1 & \text { if } \nu=2 s+2 \\
-1 & \text { if } \nu=2 s+3 \\
0 & \text { otherwise }\end{cases} \\
& c_{s+1, \nu}^{\prime}= \begin{cases}1 & \text { if } \nu=s+1 \\
-1 & \text { if } \nu=2 s+2 \\
1 & \text { if } \nu=2 s+3 \\
0 & \text { otherwise }\end{cases} \\
& c_{s+2, \nu}^{\prime}= \begin{cases}-x^{2} & \text { if } \nu=1 \\
1 & \text { if } \nu=s+2 \\
0 & \text { otherwise }\end{cases}
\end{aligned}
$$




$$
\begin{aligned}
& c_{\mu, \nu}^{\prime}=\left\{\begin{array}{ll}
-R x^{2} & \text { if } \nu=\mu-s-3 \\
\left(R^{2}+1\right) x^{2} & \text { if } \nu=\mu-s-2 \\
-R x^{2} & \text { if } \nu=\mu-s-1 \\
R^{2} & \text { if } \nu=\mu \\
0 & \text { otherwise }
\end{array} \quad \text { for } s+3 \leq \mu \leq 2 s+1\right) \\
& c_{2 s+2, \nu}^{\prime}= \begin{cases}-R x^{2} & \text { if } \nu=s-1 \\
\left(R^{2}+1\right) x^{2} & \text { if } \nu=s \\
-x^{2} & \text { if } \nu=s+1 \\
R^{2} & \text { if } \nu=2 s+2 \\
0 & \text { otherwise }\end{cases} \\
& c_{2 s+3, \nu}^{\prime}= \begin{cases}-x^{2} & \text { if } \nu=s \\
x^{2} & \text { if } \nu=s+1 \\
\frac{R^{2}}{R^{2}-1} & \text { if } \nu=2 s+3 \\
0 & \text { otherwise. }\end{cases}
\end{aligned}
$$

At this stage of our proof we may assert that

$$
\varphi_{n, k}(R)=\left(R^{n}-R^{n-2}\right) \min _{0 \leq j \leq k} X_{j, k}(R)
$$

where $X_{j, k}(R)=1-f_{j, k}(R)$ is the smallest positive root of the polynomial appearing in (4.3) and $X_{j, k}(R)$ is independent of $n$. Let $\min _{0 \leq j \leq k} X_{j, k}(R)=: 1-f_{k}(R)$ so that, in view of Corollary $2,0 \leq f_{k}(R) \leq 1$, where $f_{k}(R)$ is independent of $n$.

We will now study the asymptotic behaviour of $f_{k}(R)$. In order to do this we take the determinant in (4.3) and we

(vii) subtract its $i$-th row from its $(s+i+2)-n d$ row for $i=1,2, \ldots, s$. Then divide the $(s+i+2)$-nd row by $R$, again for $i=1,2, \ldots, s$.

(viii) Subtract the new $(s+1)$-st column from the new $(2 s+3)$-rd one. Then, for $i=1,2, \ldots, s$, we divide the $i$-th row by $R^{2}$. In the resulting determinant multiply the $(2 s+3)$-rd column by $R$. Thus

$$
\begin{aligned}
D\left(R^{m}, R^{m-1}, \ldots, R^{s+1}, R^{s}+\gamma, R^{s-1}, \ldots, R^{2}, R, 1\right) \\
\quad=R^{m^{2}-m-s-3}\left(R^{2}-1\right)^{m+1} \|\left[c_{\mu, \nu}^{\prime \prime} \|_{1 \leq \mu, \nu \leq 2 s+3}\right.
\end{aligned}
$$

where

$$
\begin{aligned}
c_{1, \nu}^{\prime \prime} & = \begin{cases}1 & \text { if } \nu=1 \\
-\frac{1}{R^{2}} & \text { if } \nu=s+2 \\
\frac{R^{2}+1}{R^{2}} & \text { if } \nu=s+3 \\
-\frac{1}{R} & \text { if } \nu=s+4 \\
0 & \text { otherwise }\end{cases} \\
c_{\mu, \nu}^{\prime \prime} & =\left\{\begin{array}{ll}
1 & \text { if } \nu=\mu \\
-\frac{1}{R} & \text { if } \nu=\mu+s+1 \\
\frac{R^{2}+1}{R} & \text { if } \nu=\mu+s+2 \\
-\frac{1}{R} & \text { if } \nu=\mu+s+3 \\
0 & \text { otherwise }
\end{array} \quad \text { (for } 2 \leq \mu \leq s\right)
\end{aligned}
$$




$$
\begin{aligned}
& c_{s+1, \nu}^{\prime \prime}= \begin{cases}1 & \text { if } \nu=s+1 \\
-1 & \text { if } \nu=2 s+2 \\
0 & \text { otherwise }\end{cases} \\
& c_{s+2, \nu}^{\prime \prime}= \begin{cases}-x^{2} & \text { if } \nu=1 \\
1 & \text { if } \nu=s+2 \\
0 & \text { otherwise }\end{cases} \\
& c_{s+3, \nu}^{\prime \prime}= \begin{cases}Y & \text { if } \nu=1 \\
-x^{2} & \text { if } \nu=2 \\
\frac{1}{R} & \text { if } \nu=s+2 \\
-\frac{1}{R} & \text { if } \nu=s+3 \\
1 & \text { if } \nu=s+4 \\
0 & \text { otherwise }\end{cases}
\end{aligned}
$$

(here, and in what follows, $\left.Y:=R\left(x^{2}-1\right)+\frac{x^{2}}{R}\right)$ and

$$
\begin{aligned}
& c_{\mu, \nu}^{\prime \prime}=\left\{\begin{array}{ll}
-x^{2} & \text { if } \nu=\mu-s-3 \\
Y & \text { if } \nu=\mu-s-2 \\
-x^{2} & \text { if } \nu=\mu-s-1 \\
1 & \text { if } \nu=\mu-1 \\
-\frac{1}{R} & \text { if } \nu=\mu \\
1 & \text { if } \nu=\mu+1 \\
0 & \text { otherwise }
\end{array} \quad \text { (for } s+4 \leq \mu \leq 2 s+1\right) \\
& c_{2 s+2, \nu}^{\prime \prime}= \begin{cases}-x^{2} & \text { if } \nu=s-1 \\
Y & \text { if } \nu=s \\
-\frac{x^{2}}{R} & \text { if } \nu=s+1 \\
1 & \text { if } \nu=2 s+1 \\
-\frac{1}{R} & \text { if } \nu=2 s+2 \\
x^{2}+1 & \text { if } \nu=2 s+3 \\
0 & \text { otherwise }\end{cases} \\
& c_{2 s+3, \nu}^{\prime \prime}= \begin{cases}-x^{2} & \text { if } \nu=s \\
x^{2} & \text { if } \nu=s+1 \\
R\left(1-x^{2}\right)+\frac{R}{R^{2}-1} \quad \text { if } \nu=2 s+3 & \text { otherwise. } \\
0 & : \quad 1\end{cases}
\end{aligned}
$$

(ix) In identity (4.5) let us make the substitution $x=1-\frac{c}{R}$. In view of Lemma 4 the roots of the polynomial appearing in (4.5) are real. Thus invoking Hurwitz's theorem we may infer that

$$
\lim _{R \rightarrow \infty} R f_{j, k}(R)=: d_{j, k}
$$


where $d_{j, k}$ is the positive root, closest to one, of the limiting polynomial

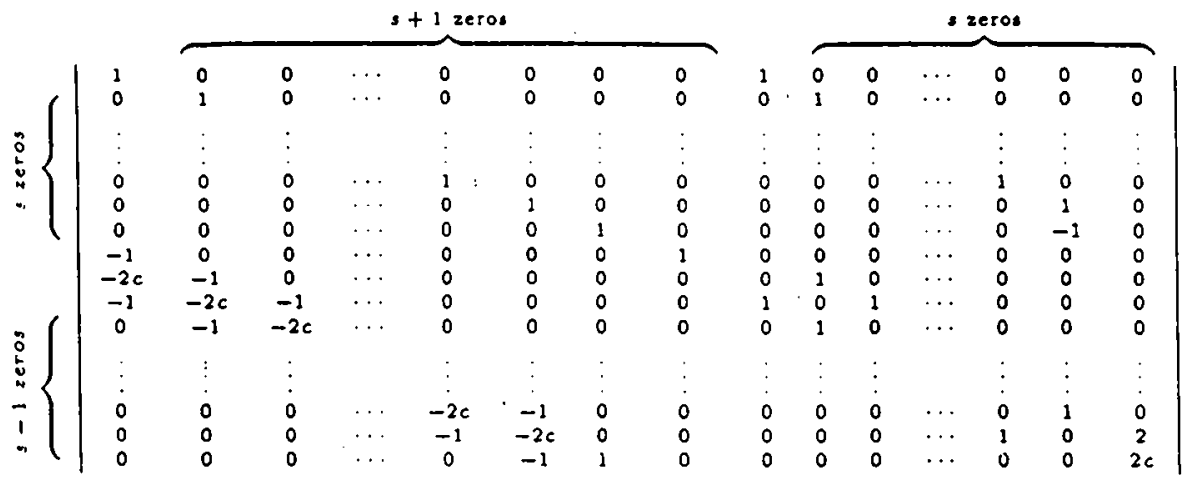

which we denote by $G(c)$. This limiting polynomial is equal to $2 c$ if $s=0$, to $4\left(c^{2}-1\right)$ if $s=1$ and to $8\left(c^{3}-2 c\right)$ if $s=2$. Our next goal is to calculate the value of $d_{j, k}$. For this we further simplify (4.6) by

(x) subtracting its $(s+l+2)$-th column from its $l$-th one for $l=1,2, \ldots, s$. We note that as regards rows number 1 to $s$ of the resulting determinant each one of them contains exactly one non-zero element and that it is 1 . We develop the determinant by these rows one after the other. Next, in the determinant of order $s+3$ so obtained we

(xi) subtract the $(s+1)$-st column from the $s$-th column. Finally expanding the determinant by its first row and then by its $(s+2)$-nd column we get

$$
G(c)=2^{k-j+1} \cdot D(c, 1, \underbrace{0, \ldots, 0}_{k-j-1 \text { zeros }}) .
$$

It is well known (see, for example, [2]) that

$$
D(c, 1, \underbrace{0, \ldots, 0}_{k-j-1 \text { zeros }})=\frac{1}{\sqrt{c^{2}-4}}\left\{\left(\frac{c+\sqrt{c^{2}-4}}{2}\right)^{k-j+2}-\left(\frac{c-\sqrt{c^{2}-4}}{2}\right)^{k-j+2}\right\} .
$$

It follows the equivalence

$$
D(c, 1, \underbrace{0, \ldots, 0}_{k-j-1 \text { zeros }})=0 \Longleftrightarrow c=2 \cos \frac{\nu \pi}{k-j+2} \quad(0<\nu<k-j+2)
$$

whence $d_{j, k}=2 \cos \frac{\pi}{k-j+2}$. So,

$$
\lim _{R \rightarrow \infty} R f_{k}(R)=\max _{0 \leq j \leq k} d_{j, k}=2 \cos \frac{\pi}{k+2} .
$$

This completes the proof of the theorem. 


\section{Concluding remarks}

In this section we present additional results which turn out to be consequences of the proof of our Theorem.

5.1 Since the quantity $\min _{0 \leq j \leq k} X_{j, k}(R)$ appearing in (4.4) is independent of $n$, we readily obtain

$$
\varphi_{n+1, k}(R)=R \varphi_{n, k}(R) \quad(n \geq 2 k+2) .
$$

This result in conjunction with (3.4) gives

$$
\varphi_{n, k}(R) \leq \varphi_{n, k-1}(R) \quad(n \geq 2 k+2) .
$$

5.2 Another interesting observation is one which links $\varphi_{n, k}(R)$ with the constant $c_{k}$ appearing in the statement of Theorem A: let us write inequality (1.6) as

$$
\frac{M_{P}(R)-M_{P}(1)}{R-1}+\frac{\varphi_{n, k}(R)}{R-1}\left|a_{k}^{i}\right| \leq \frac{R^{n}-1}{R-1}\|P\| \quad(R>1) .
$$

We will show that $\lim _{R \rightarrow 1} \frac{\varphi_{n, k}(R)}{R-1}=: \psi_{k}$ exists, at least if $n \geq 2 k+2$. Thus, provided $M_{P}^{\prime}(1)$ exists we will obtain

$$
M_{P}^{\prime}(1)+\psi_{k}\left|a_{k}\right| \leq n\|P\|
$$

It follows from (4.4) that, for $n \geq 2 k+2$,

$$
\psi_{k}=2 \lim _{R \rightarrow 1}\left(\min _{0 \leq j \leq k} X_{j, k}(R)\right)=2 \min _{0 \leq j \leq k}\left(\lim _{R \rightarrow 1} X_{j, k}(R)\right)
$$

if $\lim _{R \rightarrow 1} X_{j, k}(R)$ exists for $0 \leq j \leq k$. But if we multiply the last row of equality (4.3) by $R^{2}-1$, let $R \rightarrow 1$ in the determinant so obtained and expand the resulting determinant by its last row we see that $\lim _{R \rightarrow 1} X_{j, k}(R)=: \psi_{k}^{(j)}$ is the smallest positive root of the equation

$$
D(1, \underbrace{0,0, \ldots, 0}_{k-j \text { zeros }},-x, 2 x,-x, \underbrace{0,0, \ldots, 0}_{k-j-2 \text { zeros }})=0 .
$$

It is known from [3] that $\min _{0 \leq j \leq k} \psi_{k}^{(j)}=\psi_{k}^{(0)}$ and we infer that $\psi_{k}=c_{k}$. Hence, applying estimate (5.1) to the polynomial $P(\rho z)(\rho>0)$ we obtain the following result:

Let $P \in \mathcal{P}_{n}(n \geq 2 k+2)$ and $\rho>0$. If $M_{P}^{\prime}(\rho)$ exists, then

$$
\rho M_{P}^{\prime}(\rho)+c_{k}\left|a_{k}\right| \rho^{k} \leq n M_{P}(\rho)
$$

where $c_{k}$ is the constant defined in Theorem $A$. 


\section{References}

[1] Bernstein, S. N: Leçons sur les propiétés extrémales et la meilleure approximation des fonctions analytiques d'une variable réelle. Paris: Gauthier-Villars 1926. Reprinted in the first part of: Bernstein, S. N. and C. De La Vallée Poussin: L'approximation. New York: Chelsea 1970.

[2] Frappier, C. and M. A. Qazi: Optimal inequalities for the coefficients of polynomials of small degree. Annales Univ. Mariae Curie-Skłodowska, Sectio A: Math. 47 (1994), 18 26.

[3] Frappier, C. and M. A. Qazi: Asymptotic inequalities for polynomials with restricted coefficients. Analysis (to appear).

[4] Frappier, C.: Inequalities for polynomials with restricted coefficients. J. d'Analyse Math. 50 (1988), 143 - 157.

[5] Frappier, C., Rahman, Q. I. and St. Ruscheweyh: New inequalities for polynomials. Trans. Amer. Math. Soc. 288 (1985), 69 - 99.

[6] Gantmacher, F. R.: The Theary of Matrices. New York: Chelsea 1959.

[7] Riesz, M.: Über einen Satz des Herm Serge Bernstein. Acta Math: 40 (1916), $337-347$.

Received 02.11.1995 